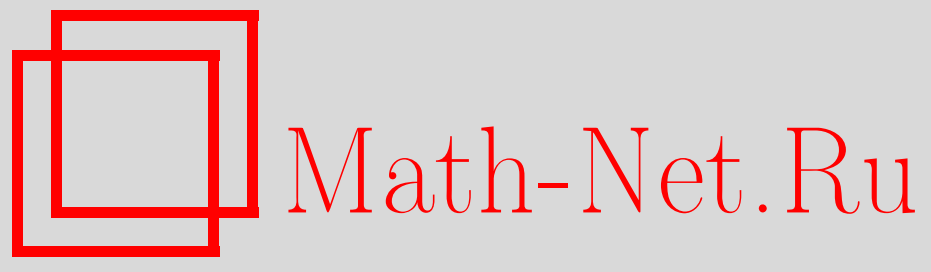

Г. Фалькович, Конформная инвариантность в гидродинамической турбулентности, УМН, 2007, том 62, выпуск 3, 193-206

DOI: https://doi.org/10.4213/rm6118

Использование Общероссийского математического портала Math-Net.Ru подразумевает, что вы прочитали и согласны с пользовательским соглашением http://www . mathnet.ru/rus/agreement

Параметры загрузки:

IP : 54.224 .187 .69

26 апреля 2023 г., 17:44:01

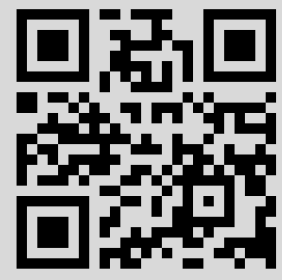




\title{
Конформная инвариантность в гидродинамической турбулентности
}

\section{Г. Фалькович}

\begin{abstract}
Этот текст написан физиком. В нем совсем нет теорем и аккуратных определений. Основное содержание составляет описание результатов численного решения уравнений гидродинамики в режиме развитой турбулентности. Вследствие ограниченных возможностей компьютеров, результаты обладают конечной (и немалой) погрешностью. Не будучи ни точными, ни строгими, эти находки, тем не менее, могут представлять интерес для математиков. Главный результат: изолинии скалярных полей (завихренности, температуры) в двумерной турбулентности относятся к классу конформно-инвариантных кривых, называемых SLE (Scramm-Loewner evolution). Это позволяет, во-первых, предсказать и обнаружить богатые семейства количественных соотношений, далеко выходящие за рамки всех предыдущих знаний о турбулентности. Во-вторых, свидетельствует о связях между понятиями, которые представлялись далекими (как уравнение Эйлера и критическая перколяция, например). В-третьих, свидетельствует о возможности получения точных аналитических результатов в статистической гидродинамике. Словом, физики обнаружили нечто неожиданное и надеются, что математики помогут это объяснить.
\end{abstract}

Библиография: 45 названий.

\section{СоДЕРЖАНИЕ}

Введение . . . . ............................................. 193

$\S 1$. Гидродинамические модели и обратные каскады ................. 195

$\S 2$. Эволюция Шрамма-Лёвнера (SLE) . . . . . . . . . . . . . . . . . . . 197

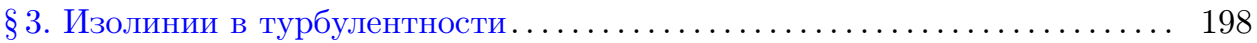

$\S 4$. Обсуждение результатов ................................ 202

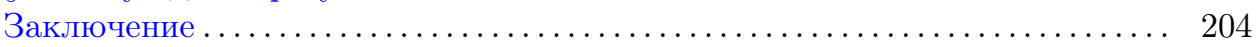

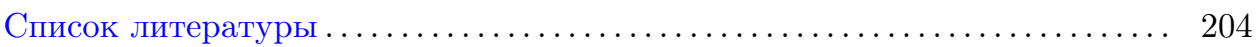

\section{Введение}

Центральная тема - симметрии статистики турбулентности. Турбулентность есть состояние сплошной среды, отклоненной далеко от равновесия. Внешние силы, ответственные за отклонение, могут быть регулярными или случайными;

(C) Г. ФАлькович, 2007 
мы ограничимся здесь случайными силами в виде конечного числа независимых гармоник Фурье с нормальным распределением. Даже в этом случае предсказать статистику, например, поля скорости, возбуждаемого такими силами, не удается из-за нелинейности уравнений гидродинамики. Мы надеемся, однако, что некоторые свойства статистики скорости не зависят от свойств возбуждающей силы. В частности, поэтому мы интересуемся симметриями. А.Н.Колмогоров в 1941 г. предположил масштабную инвариантность статистики скорости в инерционном интервале трехмерной турбулентности. Конкретно, плотность вероятности $\mathscr{P}(\delta v, r)$ разности скоростей $\delta v$, измеренной на расстоянии $r$ (много меньше масштаба внешней силы и много больше внутреннего, вязкого, масштаба), предполагалась автомодельной. Это значит, что можно найти такие число $h$ и функцию $f$ от одной переменной, что $\mathscr{P}(\delta v, r)=(\delta v)^{-1} f\left(\delta v / r^{h}\right)[1]$. Трехмерная турбулентность несжимаемой жидкости реализует пример так называемого прямого каскада, когда генерация происходит на больших, а диссипация - на малых масштабах. Как мы сейчас знаем (из экспериментов и решения нескольких модельных задач), масштабная инвариантность нарушена практически во всех известных прямых каскадах [2], [3]. На интуитивном уровне подобные нарушения представляются естественными (в духе замечания Ландау Колмогорову на семинаре в Казани в 1942 г. [2]), поскольку рассматриваются флуктуации внутри радиуса корреляции случайной внешней силы; симметрии, нарушенные силой (масштабная инвариантность, изотропия), остаются нарушенными. Мы даже научились в некоторых случаях находить семейства мартингалов (которые мы называем статистическими интегралами движения или нулевыми модами), описывающие эти нарушения [3]-[5].

Существуют, однако, предсказанные Р. Крейчнаном [6] и В. Е. Захаровым [7] случаи, когда турбулентность реализуется на масштабах, превышающих радиус корреляции внешней силы. Такие примеры принято называть обратными каскадами. Эксперименты и численное моделирование в пределах погрешности демонстрируют масштабную инвариантность статистики в обратных каскадах [8]--[12]. Объяснить или описать это свойство мы не умеем. Тем не менее, мы пытаемся сделать следующий шаг и выяснить, нельзя ли расширить симметрию до конформной инвариантности. Такая программа была предложена А. М. Поляковым [13]. Особенно заманчиво это было бы в двумерном случае, где конформная группа бесконечномерна, так что накладываемые ею ограничения позволяют зафиксировать многие характеристики статистики, как это было эффективно проделано физиками в теории поля и критических явлений [14].

Традиционно, требования конформной ковариантности накладывались на многоточечные корреляционные функции. На этом пути удалось получить аналитически несколько отрицательных результатов, доказав отсутствие конформной симметрии в турбулентности пассивного скалярного поля, переносимого случайным полем скорости с коротким временем корреляции [15], [16]. Что же касается самого поля скорости (или завихренности), описываемого уравнениями Эйлера, Навье-Стокса или другими гидродинамическими моделями, никакой информации о многоточечных корреляционных функциях не получено до сих пор ни аналитически, ни численно, ни экспериментально. Недавно появился, однако, новый язык для обсуждения статистической конформной инвариантности. Здесь предметом являются плоские случайные кривые (без самопересечений), относящиеся к семейству SLE (Scramm-Loewner evolution) [17]-[20]. Основное содержание этого короткого обзора - демонстрация того, что линии уровня завихренности в двумерном уравнении Эйлера и температу- 
ры в поверхностной квазигеострофической модели принадлежат к классу SLE, и обсуждение последствий этого. План обзора следующий: описание гидродинамических моделей и обратных каскадов в них, определение SLE и нескольких базисных фактов и гипотез, описание результатов численного моделирования из [16], [21], заключительные замечания.

\section{§1. Гидродинамические модели и обратные каскады}

Рассмотрим вещественную функцию координат и времени $a(\mathbf{r}, t)$, эволюционирующую согласно уравнению

$$
\frac{\partial a}{\partial t}+(\mathbf{v} \cdot \nabla) a=f+\nu \Delta a-\alpha a
$$

Здесь $\mathbf{r}=(x, y)$ принадлежит двумерному многообразию (плоскость, диск или двумерный тор). На этом многообразии определено несжимаемое векторное поле скорости $\mathbf{v}=(\partial \Psi / \partial y,-\partial \Psi / \partial x)$. Функция тока $\Psi$ и переносимая величина $a$ связаны линейным масштабно-инвариантным соотношением $\Psi(\mathbf{r}, t)=\int d \mathbf{r}^{\prime}\left|\mathbf{r}-\mathbf{r}^{\prime}\right|^{2-m} a\left(\mathbf{r}^{\prime}, t\right)$, где $m$ - целое число для моделей, представляющих физический интерес. То есть $а$ переносится скоростью $\mathbf{v}$, накачивается внешней силой $f$ и затухает из-за вязкости и однородного трения (например, одно). Коэффициенты вязкости и однородного трения - это соответственно $\nu$ и $\alpha$.

Выбор $m=2$ отвечает двумерному уравнению Эйлера, в этом случае псевдоскаляр $a=\operatorname{rot} \mathbf{v}=\Delta \Psi-$ это завихренность и $\Psi(\mathbf{r}, t)=\int d \mathbf{r}^{\prime} \ln \left|\mathbf{r}-\mathbf{r}^{\prime}\right| a\left(\mathbf{r}^{\prime}, t\right)$. Мы также рассмотрим $m=1$, что соответствует поверхностной квазигеострофической модели, которая описывает течения вращающейся неоднородно нагретой жидкости под воздействием архимедовой силы вблизи твердой поверхности; в этом случае $a$ - это температура [22], [23]. Представляет физический интерес также случай $m=-2$, описывающий крупномасштабные движения вращающейся мелкой воды (не рассматривается здесь) [24].

В представлении Фурье скорость выражается следующей формулой: $\mathbf{v}_{\mathbf{k}}=$ $-i\left(k_{2},-k_{1}\right) \Psi_{\mathbf{k}}=-i\left(k_{2},-k_{1}\right) k^{-m} a_{\mathbf{k}}$. Например, для тора $2 \pi \times 2 \pi$ коэффициенты Фурье $a_{\mathbf{k}}(t)=\int a(\mathbf{x}, t) e^{i(\mathbf{k} \cdot \mathbf{x})} d \mathbf{x}$ эволюционируют согласно уравнению

$$
\begin{gathered}
\frac{\partial a_{\mathbf{k}}}{\partial t}-\sum_{\mathbf{j}}[\mathbf{k}, \mathbf{j}] j^{-m} a_{\mathbf{j}} a_{\mathbf{k}-\mathbf{j}}=f_{\mathbf{k}}-\left(\alpha+\nu k^{2}\right) a_{\mathbf{k}}, \\
\mathbf{k}, \mathbf{j} \in \mathbb{Z}^{2} \backslash(0,0), \quad[\mathbf{k}, \mathbf{j}]=k_{1} j_{2}-k_{2} j_{1}, \quad k=|\mathbf{k}|, \quad j=|\mathbf{j}| .
\end{gathered}
$$

Случайная сила обычно полагается гауссовой: $d f(\mathbf{r}, t)=\sum_{\mathbf{k}} \sqrt{D(\mathbf{k})} e^{i(\mathbf{k} \cdot \mathbf{r})} d B_{\mathbf{k} t}$, где $B_{\mathbf{k} t}$ - стандартные одномерные броуновские движения, независимые для разных k. Спектральная плотность $D(\mathbf{k})$ отлична от нуля в кольце $k_{f}<k<$ $A k_{f}$, здесь $A$ - число порядка единицы. Поля $a, \mathbf{v}, \Psi$ связаны с силой нелинейным уравнением так, что их статистика, вообще говоря, негауссова.

Левая часть уравнения (2) сохраняет $L^{2}$-норму $\sum\left|a_{\mathbf{k}}\right|^{2}$ и "энергию" $E=$ $\sum\left|a_{\mathbf{k}}\right|^{2} k^{-m}$, а правая часть описывает генерацию и поглощение. Мы предполагаем существование статистически стационарного состояния, в котором 
средние $\mathrm{E}[\cdot]$ не зависят от времени. В этом случае должно выполняться

$$
\begin{gathered}
\sum_{\mathbf{k}}\left(\alpha+\nu k^{2}\right) \mathrm{E}\left[\left|a_{\mathbf{k}}\right|^{2}\right]=\sum D(\mathbf{k}) \equiv P, \\
\sum_{\mathbf{k}}\left(\alpha+\nu k^{2}\right) k^{-m} \mathrm{E}\left[\left|a_{\mathbf{k}}\right|^{2}\right]=\sum D(\mathbf{k}) k^{-m} \equiv Q .
\end{gathered}
$$

Рассмотрим предел $k_{f} \rightarrow \infty, \nu \rightarrow 0$ при фиксированных $P, Q$. Для $m>0$ естественно предположить, что основной вклад в левую сумму в (3) будут давать $k \simeq k_{\nu} \gg k_{f}$, а в левую сумму в $(4) k \simeq k_{\alpha} \ll k_{f}$. На подобных соображениях основана картина двойного каскада Крейчнана, в которой постулируется наличие двух инерционных интервалов, где доминирует нелинейный (инерционный) член уравнения (1), осуществляющий спектральную перекачку $P$ и $Q$ в прямом и обратном каскаде соответственно [6]. Рассмотрим обратный каскад, который определяется потоком $Q$. Показатели степеней можно попытаться угадать из соображений размерности. При сравнении степеней сантиметров

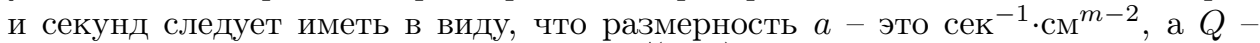
это $\mathrm{ceK}^{-3} \cdot \mathrm{cm}^{4-m}$. Тогда $k_{\alpha} \simeq\left(\alpha^{3} / Q\right)^{1 /(4-m)}$, где $\simeq$ означает, что равенство выполняется с точностью до безразмерной конечной константы (вообще говоря, зависящей от деталей статистики силы). Предполагая, что распределение в инерционном интервале обратного каскада (т.е. при $k_{\alpha} \ll k \ll k_{f}$ ) определяется потоком энергии $Q$ и волновым числом $k$, получим из соображений размерности

В $r$-представлении

$$
\mathrm{E}\left[\left|a_{\mathbf{k}}\right|^{2}\right] \simeq Q^{2 / 3} k^{(4 m-4) / 3} .
$$

$$
\mathrm{E}\left[a_{r}^{2}\right]=\sum_{\mathbf{k}} \mathrm{E}\left[\left|a_{\mathbf{k}}\right|^{2}\right]\left(1-e^{i(\mathbf{k} \cdot \mathbf{r})}\right) \simeq Q^{2 / 3} r^{(4-4 m) / 3} \propto r^{2 h} .
$$

При $m=1 \quad \mathrm{E}\left[a_{r}^{2}\right] \propto \ln \left(k_{f} r\right)$. Чуть более аккуратный способ получения индекса $h$ состоит в требовании постоянства тройного коррелятора, задающего поток энергии. Например, для уравнения Эйлера $(m=2)$ так получается равенство $\left\langle(\delta \mathbf{v} \cdot \mathbf{r})^{3}\right\rangle=3 Q r^{4} / 2$, дающее скейлинг Колмогорова-Крейчнана $h=-2 / 3$ [2], [4], [6]. Лучшее, что можно сказать о подобных соображениях, это что они подтверждаются экспериментами и численным моделированием, а именно, в пределах погрешности, обусловленной конечной длиной инерционного интервала и погрешностью измерений в эксперименте, функция распределения является инвариантной относительно глобальных (однородных) преобразований масштаба: $\mathscr{P}\left(a_{r}, r\right) \sim a_{r}^{-1} f\left(a_{r} r^{2 / 3}\right)$ для $m=2[8]-[12]$ и $\mathscr{P}\left(a_{r}, r\right) \sim$ $a_{r}^{-1} f\left(a_{r} / \ln \left(k_{f} r\right)\right)$ для $m=1$ [12], [25], [26]. Конформные преобразования осуществляют неоднородные растяжения (сохраняя при этом углы), так что конформная инвариантность может рассматриваться как локальная масштабная инвариантность. Заметим, что наши системы динамически нелокальны вследствие нелокальной связи скорости $\mathbf{v}$ и переносимого поля $a$. Мы, однако, возбуждаем систему шумом с коротким радиусом корреляции $k_{f}^{-1}$ и надеемся обнаружить локальность в статистических свойствах. Именно, пусть турбулентность в некой односвязной области $\mathscr{D} \in C$ задает семейство мер $\mu_{\mathscr{D}}\left(z_{1}, \ldots, z_{n}\right)$, зависящих от точек $z_{i} \in \mathscr{D}$ (например, распределение вероятностей для разностей скоростей в разных точках). Турбулентность, возбуждаемая накачкой с тем же $k_{f}$ в другой области $\mathscr{D}^{\prime}$, задаст другое семейство $\mu_{\mathscr{D}^{\prime}}$. Мы называем меру конформно инвариантной, если она инвариантна относительно конформного отображения $f: \mathscr{D} \rightarrow \mathscr{D}^{\prime}$, т.е. $\mu_{\mathscr{D}}\left(z_{1}, \ldots, z_{n}\right)=\mu_{\mathscr{D}^{\prime}}\left(f\left(z_{1}\right), \ldots, f\left(z_{n}\right)\right)$. Такое 
свойство выполняется для некоего замечательного класса случайных кривых, который мы сейчас кратко опишем.

\section{§ 2. Эволюция Шрамма-Лёвнера (SLE)}

Кривую без самопересечений, растущую с границы области, можно описывать конформным отображением области с кривой внутри на область без кривой. Например, в простейшем случае кривая $\gamma(t)$ стартует с вещественной оси верхней полуплоскости $H$. Здесь $t$ - параметр на кривой, не следует путать его со временем из (1). Отображение $g_{t}: H \backslash \gamma(t) \rightarrow H$ фиксируется асимптотикой $g_{t}(z) \sim z+2 t / z+\mathrm{O}\left(1 / z^{2}\right)$ на бесконечности. Если кривая касается себя, то следует определить область $K(t)$ как объединение кривой и всех точек, недостижимых из бесконечности, и рассматривать $g_{t}: H \backslash K(t) \rightarrow H$. При этом растущая крайняя точка кривой отображается в вещественную точку $\xi(t)$. В 1923 г. К. Лёвнер обнаружил, что аналитическая функция $g_{t}(z)$ и кривая $\gamma(t)$ полностью определяются положением образа крайней точки $\xi(t)$, называемой управляющей (driving) функцией [27]. Для этого следует решить замечательно простое уравнение Лёвнера:

$$
\frac{d g_{t}(z)}{d t}=2\left[g_{t}(z)-\xi(t)\right]^{-1}
$$

Почти через 80 лет О. Шрамм применил эволюцию Лёвнера к случайным плоским кривым и показал (сначала в частном случае), что мера на кривых является конформно инвариантной тогда и только тогда, когда $\xi(t)=\sqrt{\kappa} B_{t}$, где $B_{t}$ - стандартное одномерное броуновское движение [28]. Вдобавок, мера $\mu_{H}\left(\gamma ; z_{1}, z_{2}\right)$ на кривых $\gamma$, идущих из $z_{1}$ в $z_{2}$, является марковской: если разделить $\gamma$ на два отдельных сегмента: $\gamma_{1}$ от граничной точки $z_{1}$ до $z$ и $\gamma_{2}$ от $z$ до $z_{2}$, то условная мера $\mu_{H}\left(\gamma_{2} \mid \gamma_{1} ; z_{1}, z_{2}\right)=\mu_{H \backslash \gamma_{1}}\left(\gamma_{2} ; z, z_{2}\right)$. Коэффициент диффузии $\kappa$ позволяет классифицировать классы конформно инвариантных кривых, обозначаемые $\mathrm{SLE}_{\kappa}$. Такие кривые ранее встречались в физике как границы кластеров в двумерных критических явлениях, описываемых конформными теориями поля, так что SLE предоставило как новый язык общения физиков и математиков, так и весьма плодотворный формализм, активно эксплуатируемый сейчас для получения новых результатов в математике, теории поля и теории критических явлений [17]-[20], [29], [30]. В следующем параграфе мы увидим, что SLE встречается также в гидродинамике.

Здесь же приведем несколько базисных фактов (и гипотез), которые понадобятся нам в дальнейшем. При $\kappa=0 \quad \gamma$-это вертикальная прямая линия. Чем больше $\kappa$, тем кривее кривая. Она является простой (т.е. с вероятностью 1 не касается ни себя, ни вещественной оси) при $0 \leqslant \kappa<4$. Если $4 \leqslant \kappa<8$, то кривая касается себя и оси, но не заполняет пространство. В этом случае можно определить внешнюю огибающую кривой как часть, достижимую из бесконечности. Внешняя огибающая кривой из $\mathrm{SLE}_{\kappa}$ принадлежит к дуальному классу $\mathrm{SLE}_{\kappa_{*}}$ с $\kappa_{*}=16 / \kappa[31]-[33]$. Фрактальная (хаусдорфова) размерность кривой из $\mathrm{SLE}_{\kappa}$ дается формулой $D_{\kappa}=1+\kappa / 8$ для $\kappa<8$.

Среди дуальных пар, $\kappa$ и $\kappa_{*}$, одна является выделенной с точки зрения локальности. Именно, кривая из $\mathrm{SLE}_{6}$ в ограниченной области не чувствует границы, пока не достигнет ее (аккуратное определения этого свойства, называемого локальностью SLE, можно найти в [20]). Дуальная ей кривая $\mathrm{SLE}_{8 / 3}$ обладает свойством ограниченности: если исключить часть области, то статистика будет такой же, как для подмножества кривых, не проходящих через 
эту часть. Интуитивно эти свойства можно понять, рассмотрев решеточные (дискретные) модели, которые отвечают соответствующим SLE в непрерывном пределе [20], [30]. Рассмотрим, например, гексагональную решетку. Случайное блуждание по граням стартует с точки границы, на которой слева все гексагоны черные, а справа - белые, и сохраняет это свойство при движении, поворачивая вправо/влево, встретив черный/белый гексагон. SLE 6 получается из классической модели протекания (перколяции) в критическом случае, когда каждый встреченный гексагон окрашен в данный цвет с вероятностью $1 / 2$. $\mathrm{SLE}_{8 / 3}$ соответствует случайному блужданию без самопересечений, когда каждая грань посещается не более одного раза. Еще одно значение $\kappa=4$ выделено тем, что самодуально, оно соответствует так называемому гармоническому навигатору. В этом случае вероятность окраски встреченного гексагона задается гармонической функцией в области с такой границей, куда включаются также гексагоны, окрашенные по дороге (иными словами, из рассматриваемого гексагона выпускается добавочное случайное блуждание, которое окрашивает его в тот цвет границы, на который оно попадет) [20], [30], [34]. В непрерывном пределе $\mathrm{SLE}_{6}$ и $\mathrm{SLE}_{4}$ возникают также как изолинии гауссовских случайных полей. Если рассмотреть поверхность графика случайной функции двух переменных $a(x, y)$ как горный рельеф в эпоху всемирного потопа, то при поднятии уровня воды наступит момент, когда вероятность пройти из конца в конец по суше сравняется с вероятностью проплыть по воде. При этом линия берега является $\mathrm{SLE}_{6}$, если корреляции функции $a(x, y)$ убывают достаточно быстро. В частности, существует (нестрогий, но правдоподобный) критерий Харриса, утверждающий, что если $\langle a(\mathbf{r}) a(0)\rangle \sim r^{-2 h}$ и $h<3 / 4$, то эквивалентность линий уровня и $\mathrm{SLE}_{6}$ нарушается [35]. Что же касается $\mathrm{SLE}_{4}$, то в этот класс попадают линий уровня гауссова случайного поля с $\langle a(\mathbf{r}) a(0)\rangle \sim \ln r$ [34], [36], [37]. Какое отношение все это имеет к гидродинамической турбулентности? Единственное, в чем мы действительно уверены, так это в негауссовости статистики турбулентности (из-за наличия тройного коррелятора, задающего поток).

\section{§ 3. Изолинии в турбулентности}

На рис. 1 показана линия нулевой завихренности, полученная при численном моделировании уравнения (1) с $m=2$ на торе (т.е. двумерного уравнения Навье-Стокса с периодическими граничными условиями и добавленными однородным трением и внешней силой). Детали численного счета могут быть найдены в [10], [21]. Масштаб накачки $l_{f}=2 \pi / k_{f}=0.05$. Видно, что кривая обладает фрактальной структурой на масштабах, превышающих $l_{f}$, т.е. в области обратного каскада. Действительно, длина кривой $P$ растет нелинейно с расстоянием между началом и концом $L$ [21]. Более того, показатели, характеризующие рост для кривой и ее внешнего периметра, в пределах погрешности близки к значениям размерностей $7 / 4,4 / 3$ для дуальной пары $\mathrm{SLE}_{6}$ и $\mathrm{SLE}_{8 / 3}$ (исторически, размерность $4 / 3$ для внешней огибающей изолинии была сначала угадана, исходя из скейлинга Колмогорова-Крейчнана, что стимулировало поиск SLE в турбулентности [21]). Опишем кратко, как мы идентифицировали кандидата на роль кривой из класса SLE в верхней полуплоскости и определяли управляющую функцию $\xi(t)$. Сначала мы провели произвольным образом прямую и назначили ее вещественной осью (мы проверили, что сдвиги и повороты оси не повлияли на результаты). Стартуя с точки пересечения нулевой изолинии и оси, мы двигаемся вдоль линии (или вдоль оси, когда снова пересекаем ее), сохраняя ориентацию, т.е. имея положительную завихренность 


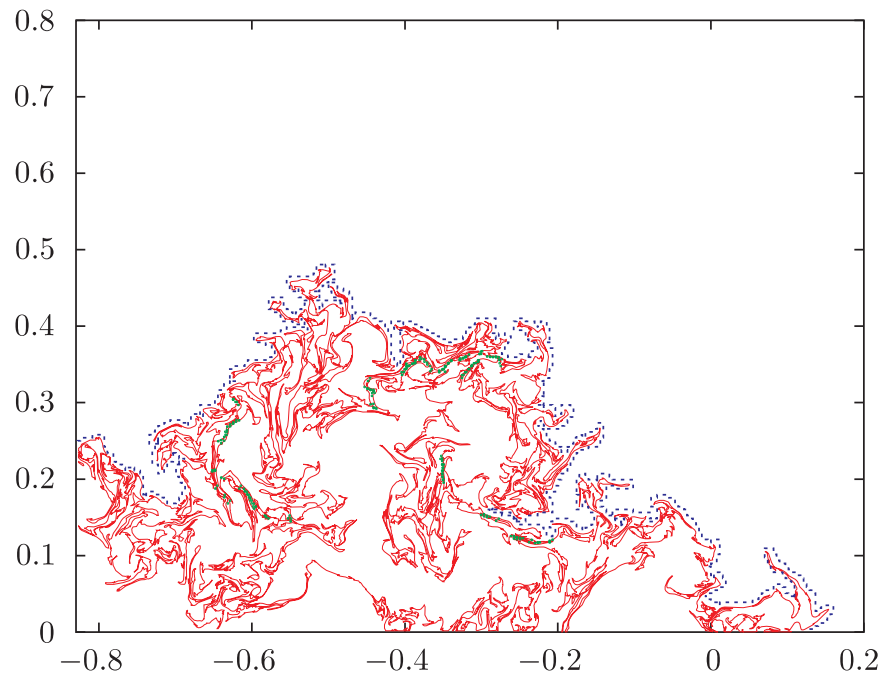

Рис. 1. Кусок линии нулевой завихренности. Пунктир - внешняя огибающая. Рисунок адаптирован из [21].

всегда справа. Такая процедура, строго говоря, хорошо воспроизводит статистику только в случае локальности, но мы как раз и ожидали (и получили!) $\kappa \approx 6$. Мы затем разбили нашу кривую на маленькие прямые сегменты и аппроксимировали семейство конформных отображений $g_{t}(z)$ дискретным набором стандартных конформных отображений, последовательно поглощающих по одному сегменту [21], [38]. В результате получается набор "времен" $t_{i}$ и значений $\xi_{i}$. Таким образом была определена управляющая функция $\xi(t)$. Осталось лишь проверить, насколько хорошо эта функция соответствует броуновскому движению (тест Шрамма). Данные, полученные в результате анализа 1.607 кривых, представлены треугольниками с основаниями внизу на рис. 2 (а). Видно, что среднее по ансамблю $\left\langle\xi(t)^{2}\right\rangle$ действительно растет линейно со временем и что $\kappa$ близко к 6 с точностью $5 \%$. Вдобавок, верхняя вставка показывает, что функция распределения величины $\xi(t) / \sqrt{\kappa t}$ близка к стандартному гауссову распределению для всех времен $t$, превышающих $l_{f}^{2} / \kappa$. Таким образом, в пределах нашей точности статистика нулевых изолиний завихренности в обратном каскаде двумерной турбулентности неотличима от $\mathrm{SLE}_{6}$. Заметим, что поле завихренности имеет $h=2 / 3<3 / 4$, т.е. критерий Харриса нарушен. Однако же, наше поле имеет негауссову статистику (хотя функция распределения на глаз и неотличима от гауссовой, отклонения измеримы, включая тройной коррелятор [8]-[12]). Треугольники с основаниями вверху на нижней вставке получены для изолиний гауссова поля, имеющего точно такой же второй момент, как и завихренность (приготовленного хаотизацией фаз коэффициентов Фурье). В этом случае, как видно, нашей точности достаточно, чтобы убедиться, что нет никакого соответствия SLE вообще и $\mathrm{SLE}_{6}$ в частности. Действительно, $\mathrm{E}\left[\xi^{2}\right] / t \equiv\left\langle\xi^{2}\right\rangle / t$ непостоянен и выходит на предельное значение, близкое к 6 , только для расстояний, больших $2 \pi / k_{\alpha}$, где обратный каскад подавлен линейным затуханием и поле некоррелировано. Цветные рисунки 1 и 2 можно найти на странице http://www.weizmann.ac.il/home/fnfal/. 


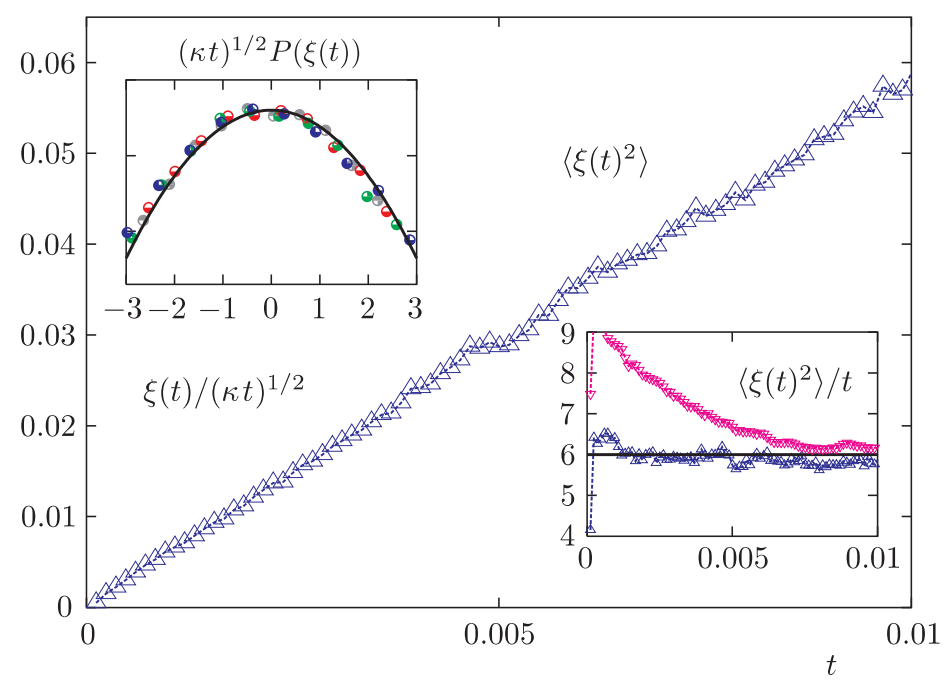

(a)

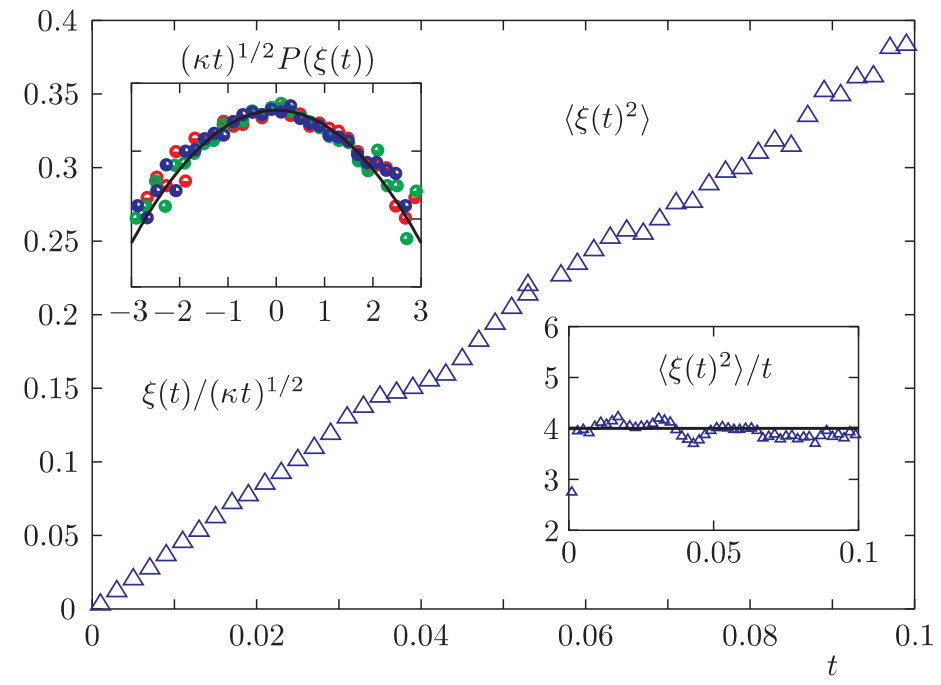

(b)

Рис. 2. Демонстрация конформной инвариантности изолиний завихренности в уравнении Эйлера (рис. (а)) и температуры в квазигеострофической модели (рис. (b)). Управляющая функция является броуновским движением с $\kappa=6 \pm 0.3$ (рис. (а), [21]) и с $\kappa=4 \pm 0.2$ (рис. (b), [16]). На правой (нижней) вставке на рис. (а) треугольникам с основаниями внизу соответствуют завихренности, а с основаниями вверху - гауссово поле с тем же вторым моментом. Левые (верхние) вставки: плотность вероятности функции $\xi(t) / \sqrt{\kappa t}$ для четырех различных времен $t=0.0012,0.003,0.006,0.009$ (рис. (а)) и $t=0.02,0.04,0.08$ (рис. (b)), сплошные линии - гауссово распределение $g(x)=(2 \pi)^{-1 / 2} \exp \left(-x^{2} / 2\right)$. 


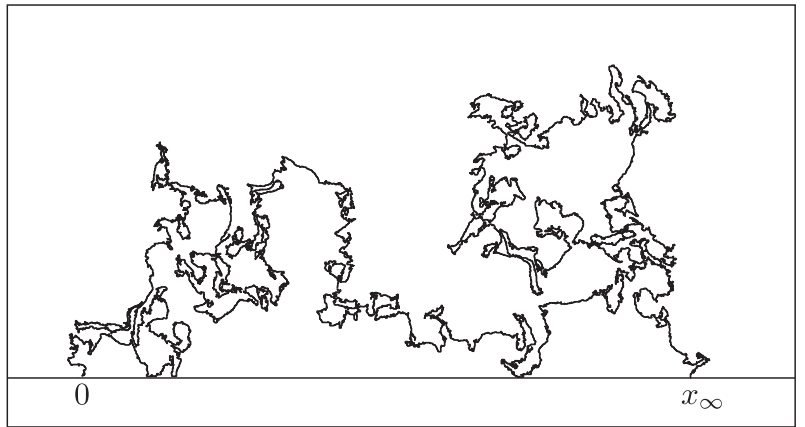

(a)

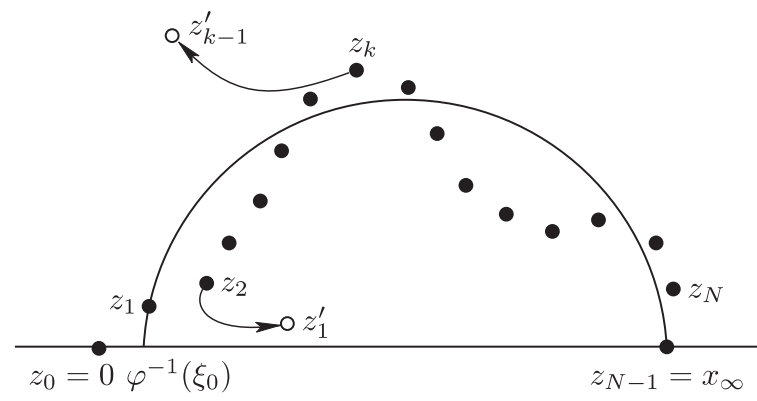

(b)

Рис. 3. (а) Часть изолинии температуры как кандидат в SLE. (b) Алгоритм извлечения ведущей функции. Рисунок адаптирован из [16].

Установленное соответствие между изолиниями завихренности и протеканием позволяет, в частности, делать аналитические предсказания по поводу статистики линий и вихрей в двумерной турбулентности. Линии нулевой завихренности являются границами кластеров вихрей. Взятые из теории протекания утверждения, что плотность вероятности кластера (материка) убывает с ростом площади как $s^{-96 / 91}$, а с ростом длины береговой линии как $P^{-8 / 7}$, подтверждаются для турбулентности [21]. Более того, SLE позволяет проводить точные вычисления вероятностей пересечения изолиний с различными фигурами (треугольниками, прямоугольниками и т.д.). Эти вероятности определяются уравнениями второго порядка, решения которых выражаются через гипергеометрические функции. Чудесным образом, получающиеся результаты совпадают с данными численного эксперимента [21]. Стоит подчеркнуть, что до этого самое большее, на что можно было надеяться в теории турбулентности, - это предсказать число (обычно, степень) из соображений размерности. С помощью нового подхода впервые удалось предсказать целые нетривиальные функции. Радует также возможность делать точные утверждения, а не по порядку величины. Главное же, турбулентность в области обратного каскада, по-видимому, эффективно описывается уравнением Эйлера, так что описанные здесь результаты намекают на некие свойства этого фундаментального уравнения, которые нам пока непонятны.

Опишем теперь вкратце результаты для поверхностной квазигеострофической модели, $m=1$. В этом случае линия нулевой температуры пересекает 
прямую линию редко, так что мы просто выбираем в качестве кандидатов в SLE участки кривых, пересекающие прямую на расстоянии от начальной точки, много большем $2 \pi / k_{\alpha}$. Пример показан на рис. 3 (а). Такая процедура выбора самосогласована для $\kappa \leqslant 4$, что мы и получим. Чтобы извлечь ведущую функцию кривой в верхней полуплоскости, идущей из 0 в $x_{\infty}$, следует теперь решать уравнение $\partial_{t} g_{t}=2 /\left\{\varphi^{\prime}\left(g_{t}\right)\left[\varphi\left(g_{t}\right)-\xi_{t}\right]\right\}$, где $\varphi(z)=x_{\infty} z /\left(x_{\infty}-z\right)$. Это уравнение на $g_{t}$ может быть решено для постоянной $\xi$ :

$$
G_{t, \xi}(z)=x_{\infty} \frac{\eta x_{\infty}\left(x_{\infty}-z\right)+\left[x_{\infty}^{4}(z-\eta)^{2}+4 t\left(x_{\infty}-z\right)^{2}\left(x_{\infty}-\eta\right)^{2}\right]^{1 / 2}}{x_{\infty}^{2}\left(x_{\infty}-z\right)+\left[x_{\infty}^{4}(z-\eta)^{2}+4 t\left(x_{\infty}-z\right)^{2}\left(x_{\infty}-\eta\right)^{2}\right]^{1 / 2}},
$$

где $\eta=\varphi^{-1}(\xi)$. В этом случае кривая - это полуокружность, соединяющая $\eta$ и $x_{\infty}$. Мы разбиваем интервал $[0, T]$ на маленькие отрезки $\left[t_{n}, t_{n+1}\right)$ с $t_{0}=0, t_{N+1}=T$, на которых ведущая функция может считаться постоянной, $\xi_{n}=\xi\left(t_{n}\right)$. Отображение $g_{t}$ находится как композиция $G_{t_{N}-t_{N-1}, \xi_{N-1}} \circ$ $\cdots \circ G_{t_{1}}, \xi_{0}$. С помощью такой дискретной процедуры возможно определить набор $\xi_{n}=\xi\left(t_{n}\right)$, исходя из кривой, аппроксимированной конечным набором точек $\left\{z_{0}, z_{1}, \ldots, z_{N+1}\right\}$, где $z_{0}=0$ и $z_{N+1}=x_{\infty}$. На первом шаге находится полуокружность, проходящая через $x_{\infty}$ и $z_{1}$ [см. рис. $\left.3(\mathrm{~b})\right]$. Это дает значения

$$
\eta_{0}=\varphi^{-1}\left(\xi_{0}\right)=\frac{\operatorname{Re} z_{1} x_{\infty}-\left(\operatorname{Re} z_{1}\right)^{2}-\left(\operatorname{Im} z_{1}\right)^{2}}{x_{\infty}-\operatorname{Re} z_{1}}
$$

и

$$
t_{1}=\frac{\left(\operatorname{Im} z_{1}\right)^{2} x_{\infty}^{4}}{4\left[\left(\operatorname{Re} z_{1}-x_{\infty}\right)^{2}+\left(\operatorname{Im} z_{1}\right)^{2}\right]^{2}} .
$$

Отображение $G_{t_{1}, \xi_{0}}$ затем переводит точки $z_{k}$ в новую последовательность, которая короче на один элемент: $z_{k}^{\prime}=G_{t_{1}, \xi_{0}}\left(z_{k+1}\right)$ с $k=1, \ldots, N$. Итерация этой процедуры определяет два набора $t_{k}$ и $\xi_{k}$, которые и аппроксимируют ведущую функцию. Процедура сначала была проверена на ансамбле кривых, порожденных случайным блужданием без самопересечений, где она выдала правильное значение $\kappa=8 / 3$ с погрешностью менее $5 \%$. Применение этой процедуры для квазигеострофической модели выдает ансамбль $\xi(t)$, чья статистика сходится при $l_{f}^{2} \lesssim \kappa t \lesssim 2 \pi / k_{\alpha}$ к гауссовой с $\left\langle\xi^{2}(t)\right\rangle=\kappa t$ и $\kappa=4 \pm 0.2$, как показано на рис. $2(\mathrm{~b})$. Мы заключаем, что в пределах погрешности изолинии температуры в квазигеострофической модели ведут себя локально как кривые из класса $\mathrm{SLE}_{4}$. Стоит заметить, что поле температуры существенно негауссово [12], [16] и совершенно непонятно, как оно может иметь изолинии с такой же статистикой, как свободное гауссово поле с таким же вторым моментом.

Заметим, что если контур $z(l)$ принадлежит классу $\mathrm{SLE}_{\kappa}$, то единичный касательный вектор $z_{l}$ обладает гауссовой статистикой (со вторым моментом, пропорциональным логарифму длины контура). Эти свойства также были обнаружены как для линий завихренности, так и температуры для обеих наших моделей (не опубликовано).

\section{§4. Обсуждение результатов}

Здесь приведено несколько несвязанных между собой замечаний о математических структурах, которые могут быть ответственны за конформную инвариантность в гидродинамической турбулентности.

Возможно, конформную инвариантность некоторых статистических свойств турбулентности удастся объяснить, анализируя алгебраическую структуру 
уравнений гидродинамики. Если задать (локально) $z=x+i y$, то генераторы конформных преобразований $\ell_{n}=-z^{n+1} \partial_{z}$ образуют алгебру, определяемую коммутатором $\left[\ell_{n}, \ell_{n^{\prime}}\right]=\left(n-n^{\prime}\right) \ell_{n+n^{\prime}}$. Изменение меры при конформных преобразованиях $z \rightarrow z+\varepsilon(z)$ задается тензором энергии-импульса $T(z)$, так что среднее от любой величины $X$ меняется на $\delta \mathrm{E}[X]=(2 \pi i)^{-1} \oint_{C} d z \varepsilon(z) \mathrm{E}[T(z) X]$, где контур $C$ должен охватывать все точки, представленные в $X$. Разложение тензора энергии-импульса $T=\sum z^{-n-2} L_{n}$ дает операторы алгебры Вирасоро, которые действуют в гильбертовом пространстве и удовлетворяют коммутационным соотношениям

$$
\left[L_{n}, L_{n^{\prime}}\right]=\left(n-n^{\prime}\right) L_{n+n^{\prime}}+\frac{c}{12} n\left(n^{2}-1\right) \delta_{n,-n^{\prime}} .
$$

Принято говорить, что алгебра Вирасоро операторов $L_{n}$ является центральным расширением алгебры операторов $\ell_{n}$. Параметр $c$ называется центральным зарядом, является мерой числа внутренних степеней свободы и описывает реакцию системы на граничные условия [39]. Центральный заряд выражается через коэффициент диффузии SLE формулой $c=(8-3 \kappa)(\kappa-6) /(2 \kappa)$ и также классифицирует модели (с точностью до дуальности), например, $\mathrm{SLE}_{6}$ и $\mathrm{SLE}_{8 / 3}$ соответствуют $c=0, \mathrm{aLE}_{4}$ соответствует $c=1$ [29], [30].

Течения же несжимаемой жидкости в области $\Sigma$ соответствуют куда более широкой алгебре Ли $\operatorname{SDiff}(\Sigma)$, генерируемой бездивергентными векторными полями $(\partial \Psi / \partial y) \partial_{x}-(\partial \Psi / \partial x) \partial_{y}$. Для плоскости в качестве базиса можно выбрать $\Psi_{i}^{j}=y^{j+1} x^{i+j+1}, i, j \in \mathbb{Z}$, при этом скобка Пуассона задается формулой

$$
\left[\Psi_{i}^{j}, \Psi_{i^{\prime}}^{j^{\prime}}\right]=\left[i\left(j^{\prime}+1\right)-j^{\prime}(j+1)\right] \Psi_{i+i^{\prime}}^{j+j^{\prime}} .
$$

Конформная алгебра Вирасоро (при $c=0$ ) является подмножеством, генерируемым $\Psi_{i}^{n i}$ с любым фиксированным целым $n$, включая нуль. Для центрального расширения необходимо существование гармонических 1-форм (имеющих нулевой ротор, но не представимых глобально как градиент скаляра) [40]. На поверхности рода $g$ имеется $2 g$ таких форм. Например, для тора соответствующая алгебра Ли имеет структурные константы $C_{\mathbf{k j}}^{\mathbf{i}}=[\mathbf{k}, \mathbf{j}] \delta_{\mathbf{k}+\mathbf{j}-\mathbf{i}}[41]$, [42]. Для тригонометрического базиса генераторы алгебры Вирасоро (с центральным расширением) можно построить в виде ряда [40]. Согласно Арнольду, эволюция течений описывается уравнением геодезических на бесконечномерном римановом многообразии, состоящем из диффеоморфизмов области течения, сохраняющих элемент площади. Различные модели (в нашем случае разные $m$ ) различаются формой энергии, которая задает метрику аналогично тензору инерции для движения твердого тела [41], [42]. На торе уравнение движения без внешней силы и диссипации имеет вид уравнения $\partial a_{\mathbf{k}} / \partial t=$ $\sum_{\mathbf{j}} j^{-m}[\mathbf{k}, \mathbf{j}] a_{\mathbf{j}} a_{\mathbf{k}-\mathbf{j}}=\sum_{\mathbf{i j l}} \alpha^{\mathbf{i j}} C_{\mathbf{k j}}^{\mathbf{l}} a_{\mathbf{i}} a_{\mathbf{l}}$ на дуальной алгебре Ли, где только обратный тензор инерции $\alpha^{\mathbf{i j}}=j^{-m} \delta_{\mathbf{i}+\mathbf{j}}$ зависит от модели. Остается понять, действительно ли стационарная мера на римановом многообразии, порождаемая мелкомасштабной случайной силой и движением по геодезическим, является конформно инвариантной, и каким образом тензор инерции задает центральный заряд.

Возможно, впрочем, что статистика поля $a(\mathbf{x})$ не является в целом конформно инвариантной (например, корреляционные функции $\left\langle a\left(\mathbf{x}_{1}\right) \ldots a\left(\mathbf{x}_{n}\right)\right\rangle$ могут не иметь вида, предписанного в конформной теории поля [39]). Мы имеем 
дело с неравновесной системой, и нет никаких оснований ожидать, что распределение вероятностей имеет гиббсовский вид $\exp [-H\{a(\mathbf{x})\} / T]$ с гамильтонианом $H$, который является интегралом от плотности, локально зависящей от $a$ (что приводит к конформной инвариантности в критических явлениях). Возможно, что только статистика изолиний является инвариантной, так что, например, вероятность того, что одна линия проходит через окрестности точек $\mathbf{x}_{1}, \ldots, \mathbf{x}_{n}$, зависит от координат как корреляционная функция в конформной теории поля (на подобную мысль наводит чтение работы [43], где строится тензор энергии-импульса для $\left.\mathrm{SLE}_{8 / 3}\right)$. В этом случае стоит изучать движение изолиний, которое описывается условием $a(z(t), t)=$ const. Это задает функцию $z(t, l)$, где $l$ - это координата вдоль изолинии, а $t$ - это время. Движение задается уравнением $\operatorname{Im}\left(\bar{z}_{t} z_{l}\right)=v_{n}=\partial \Psi / \partial l-f \nabla a /|\nabla a|^{2}$. Как видим, внешняя сила добавила коротко-коррелированную сжимаемую часть к полю скорости изолиний. При рассмотрении статистики изолиний, видимо, следует полагать, что экстремумы $a(\mathbf{x})$ являются изолированными точками, а сила $f$ не производит седловых точек (т.е. изолинии не пересекаются).

Если контур $z(t, l)$ действительно принадлежат классу $\mathrm{SLE}_{\kappa}$, то единичный касательный вектор $z_{l}$ обладает гауссовой статистикой [28], [34]. Эволюцию контуров естественно описывать конформными отображениями, зависящими от времени. Можно надеяться, что гауссовость статистики $z_{l}$ связана с интегрируемостью эволюции конформных отображений, соответствующих гидродинамическим течениям, подобно интегрируемости уравнений, описывающих лапласовский рост границ (течение невязкой жидкости, проталкиваемой сквозь вязкую [44], [45]).

Неясно, как движение в поле скорости, имеющем как коротко-, так и длиннокоррелированную части, приводит к конформной инвариантности меры и что определяет выбор $\kappa$ или $c$. Представляется, что род поверхности $\Sigma$, на которой физически происходит движение, не играет роли, поскольку однородное трение останавливает каскад на масштабах, существенно меньших размера системы. Иными словами, мы верим, что численное моделирование на торе и лабораторный эксперимент на диске дадут одинаковое значение $c$. Скорее, нетривиальная топология и возможность центрального расширения возникают из-за необходимости стока для самого существования стационарного каскада, что должно приводить к выколотым точкам при отображении на компактные многообразия (эта гипотеза сформулирована совместно с К. Гаведским). Возможно также, что ненулевой заряд $c=1$ для $m=1$ связан с неаналитичностью при $k=0$, в таком случае можно было бы ожидать $c=0$ для $m=-2,0,4$.

\section{Заключение}

Выше были приведены экспериментальные свидетельства того, что линии уровня завихренности в двумерном уравнении Эйлера и температуры в поверхностной квазигеострофической модели принадлежат к SLE. Стоит подчеркнуть, что и завихренность и температура имеют негауссовы статистики, однако принадлежность изолиний к SLE означает существование локальных полей с чисто гауссовой статистикой и намекает на возможную интегрируемость двумерных уравнений гидродинамики.

Я благодарен С. Б. Куксину, инициировавшему этот обзор, а также Н. Макарову и К. М. Ханину, прочитавшим первый вариант рукописи и сделавшим множество полезных замечаний. 


\section{Список литературы}

[1] А.Н. Колмогоров, “Локальная структура турбулентности несжимаемой вязкой жидкости при очень больших числах Рейнольдса", Докл. АH CCCP, 30:4 (1941), 9-13; англ. пер.: А. N. Kolmogoroff, "The local structure of turbulence in incompressible viscous fluid for very large Reynolds' numbers", C. R. Acad. Sci. URSS, 30 (1941), 301-305.

[2] U. Frisch, Turbulence. The legacy of A. N. Kolmogorov, Cambridge Univ. Press, Cambridge, 1995; русск. пер. У. Фриш, Турбулентность. Наследие Колмогорова, ФАЗИС, М., 1998.

[3] G. Falkovich, K. R. Sreenivasan, "Lessons from hydrodynamic turbulence", Phys. Today, 59:4 (2006), 43-49.

[4] G. Falkovich, K. Gawędzki, M. Vergassola, "Particles and fields in fluid turbulence", Rev. Modern Phys., 73:4 (2001), 913-975.

[5] B. I. Shraiman, E. D. Siggia, "Scalar turbulence", Nature, 405 (2000), 639-646.

[6] R. H. Kraichnan, "Inertial ranges in two-dimensional turbulence", Phys. Fluids, 10:7 (1967), 1417-1423.

[7] В.Е. Захаров, Волны в нелинейных средах с дисперсией, Дис. ... канд. физ.матем. наук, Институт ядерной физики, Новосибирск, 1967.

[8] H. Kellay, W. Goldburg, "Two-dimensional turbulence: a review of some recent experiments", Rep. Progr. Phys., 65:5 (2002), 845-894.

[9] S. Chen, R. E. Ecke, G. L. Eyink, M. Rivera, M. Wan, Z. Xiao, "Physical mechanism of the two-dimensional inverse energy cascade", Phys. Rev. Lett., 96:8 (2006), 084502.

[10] G. Boffetta, A. Celani, M. Vergassola, "Inverse energy cascade in two-dimensional turbulence: Deviations from Gaussian behavior", Phys. Rev. E, 61:1 (2000), R29R32.

[11] P. Tabeling, "Two-dimensional turbulence: a physicist approach", Phys. Rep., 362:1 (2002), 1-62.

[12] A. Celani, M. Cencini, A. Mazzino, M. Vergassola, "Active and passive fields face to face", New J. Phys., 6:72 (2004), 1-35.

[13] A. M. Polyakov, "The theory of turbulence in two dimensions", Nuclear Phys. B, 396:2-3 (1993), 367-385.

[14] A. A. Belavin, A. M. Polyakov, A. A. Zamolodchikov, "Infinite conformal symmetry in two-dimensional quantum field theory", Nuclear Phys. B, 241:2 (1984), 333-380.

[15] G. Falkovich, A. Fouxon, "Anomalous scaling of a passive scalar in turbulence and equilibrium", Phys. Rev. Lett., 94:21 (2005), 214502.

[16] D. Bernard, G. Boffetta, A. Celani, G. Falkovich, "Inverse turbulent cascades and conformally invariant curves", Phys. Rev. Lett., 98:2 (2006), 024501.

[17] G. Lawler, O. Schramm, W. Werner, "Values of Brownian intersection exponents. I: Half-plane exponents", Acta Math., 187:2 (2001), 237-273; "Values of Brownian intersection exponents. II: Plane exponents", Acta Math., 187:2 (2001), 275-308.

[18] G. Lawler, O. Schramm, W. Werner, "Values of Brownian intersection exponents. III: Two-sided exponents", Ann. Inst. H. Poincaré Probab. Statist., 38:1 (2002), 109-123.

[19] G. Lawler, O. Schramm, W. Werner, "Conformal restriction: The chordal case", J. Amer. Math. Soc., 16:4 (2003), 917-955.

[20] G.F. Lawler, Conformally invariant processes in the plane, Math. Surveys Monogr., 114, Amer. Math. Soc., Providence, RI, 2005.

[21] D. Bernard, G. Boffetta, A. Celani, G. Falkovich, "Conformal invariance in twodimensional turbulence", Nature Physics, 2:2 (2006), 124-128.

[22] I. M. Held, R. T. Pierrehumbert, S. T. Garner, K. L. Swanson, "Surface quasigeostrophic dynamics", J. Fluid Mech., 282 (1995), 1-20. 
[23] R. T. Pierrehumbert, I. M. Held, K. L. Swanson, "Spectra of local and nonlocal twodimensional turbulence", Chaos Solitons Fractals, 4:6 (1994), 1111-1116.

[24] V. Larichev, J. McWilliams, "Weakly decaying turbulence in an equivalent-barotropic fluid", Phys. Fluids A, 3:5 (1991), 938-950.

[25] N. Schorghofer, "Energy spectra of steady two-dimensional turbulent flows", Phys. Rev. E, 61:6 (2000), 6572-6577.

[26] K. S. Smith, G. Boccaletti, C.C. Henning, I. Marinov, C. Y. Tam, I. M. Held, G. K. Vallis, "Turbulent diffusion in the geostrophic inverse cascade", J. Fluid Mech., 469 (2002), 13-48.

[27] K. Löwner, "Untersuchungen über schlichte konforme Abbildungen des Einheitskreises. I", Math. Ann., 89:1-2 (1923), 103-121.

[28] O. Schramm, "Scaling limits of loop-erased random walks and uniform spanning trees", Israel J. Math., 118 (2000), 221-288.

[29] J. Cardy, "SLE for theoretical physicists", Ann. Physics, 318:1 (2005), 81-118.

[30] M. Bauer, D. Bernard, "2D growth processes: SLE and Loewner chains", Phys. Rep., 432:3-4 (2006), 115-221.

[31] H. Saleur, B. Duplantier, "Exact determination of the percolation hull exponent in two dimensions", Phys. Rev. Lett., 58:22 (1987), 2325-2328.

[32] V. Beffara, The dimension of the SLE curves, arXiv: math.PR/0211322.

[33] B. Duplantier, "Conformally invariant fractals and potential theory", Phys. Rev. Lett., 84:7 (2000), 1363-1367.

[34] O. Schramm, S. Sheffield, "Harmonic explorer and its convergence to SLE4", Ann. Probab., 33:6 (2005), 2127-2148.

[35] A. Weinrib, "Long-correlated percolation", Phys. Rev. B, 29:1 (1984), 387-395.

[36] J. Cardy, R. Ziff, "Exact results for the universal area distribution of clusters in percolation, Ising and Potts models", J. Statist. Phys., 110:1-2 (2003), 1-33.

[37] J. Cardy, "Linking numbers for self-avoiding walks and percolation: application to the spin quantum Hall transition", Phys. Rev. Lett., 84:16 (2000), 3507-3510.

[38] D. Marshall, S. Rohde, Convergence of the Zipper algorithm for conformal mapping, arXiv: math/0605532.

[39] P. Di Francesco, P. Mathieu, D. Sénéchal, Conformal field theory, Grad. Texts Contemp. Phys., Springer-Verlag, New York, 1997.

[40] E. Sezgin, Area-preserving diffeomorphisms, $w_{\infty}$ algebras and $w_{\infty}$ gravity, arXiv: hep-th/9202086.

[41] В. И. Арнольд, Математические методы классической механики, 2-е изд., Наука, М., 1979; англ. пер.: V. I. Arnold, "Mathematical methods of classical mechanics", Grad. Texts in Math., 60, Springer-Verlag, New York, 1989.

[42] V. I. Arnold, B. A. Khesin, Topological methods in hydrodynamics, Appl. Math. Sci., 125, Springer-Verlag, New York, 1998.

[43] B. Doyon, V. Riva, J. Cardy, "Identification of the stress-energy tensor through conformal restriction in SLE and related processes", Comm. Math. Phys., 268:3 (2006), $687-716$.

[44] M. Mineev-Weinstein, P. Wiegmann, A. Zabrodin, "Integrable structure of interface dynamics", Phys. Rev. Lett., 84:22 (2000), 5106-5109.

[45] A. Zabrodin, Growth processes related to the dispersionless Lax equations, arXiv: math-ph/0609023.

Г. Фалькович (G. Falkovich)

Институт Вейцмана, Реховот, Израиль

E-mail: gregory.falkovich@weizmann.ac.il
Поступила в редакцию 05.10 .2006 